\section{Measuring the Equation of State with Lasers}

\author{
D. Batani', M. Koenig ${ }^{2}$, T. Löwer ${ }^{3}$, A. Benuzzi², S. Bossi \\ 'Dipartimento di Fisica, Universita degli Studi di Milano \\ 'Laboratoire pour I'Utilisation des Lasers Intenses, Ecole Polytechnique, Palaiseau \\ ${ }^{3}$ Max-Planck-Institut für Quantenoptik, Garching
}

Optimised target design allows the laser generation of high-quality shock waves for measuring the equations of state of matter at high pressures using existing laser facilities.

Despite the fact that equations of state of matter at high pressures are of great interest for several fields of modern physics, notably astrophysics and inertial confinement fusion, models for the behaviour of materials at pressures above $10 \mathrm{Mbar}$ have not been validated experimentally. Moreover, the regime of applicability of the limiting case of the Thomas-FermiDirac model which is expected to describe equations of state at extremely high pressure remains unknown.

One of the main reasons for this situation is that experimentally determined equations of states are sparse for high pressures owing to the difficulty of producing very high pressure while measuring the relevant parameters. Pressures up to 1 Mbar can be obtained with static methods using high-pressure cells equipped with diamond anvils; higher pressures can be only obtained dynamically for short times by generating shock waves in a material. A few equations of state measurements in the tens of Mbar domain have been performed using nuclear explosions. In view of their cost and relatively poor reproducibility, the

Dimitri Batani received a laurea in physics from the University of Milan in 1986, and a Ph.D. in 1990 from the University of Pisa. He joined the University of Milan's Physics Department as a research scientist after spending a year as a postdoc at LULI.

Michel Koenig obtained a DEA in theoretical physics from the Université Pierre et Marie Curie, Paris, in 1979. He worked at the Commissariat de l'Energie Atomique in 198-86 where he obtained a Ph.D. He has been a member of the research staff at LULI since 1988.

Thorsten Löwer received a Ph.D. in 1994 while working at the Max-Planck Institut für Quantenoptik, Garching, where he is now a postdoc.

Alessandra Benuzzi obtained a laurea from the University of Milan in 1995 and is presently studying for a Ph.D. at the Ecole Polytechnique, Palaiseau.

Simone Bossi obtained a laurea from the University of Milan in early-1995 where he is a SIF-ENEA research fellow after spending a year at the University of Essex. environmental impact and the efforts to limit nuclear proliferation, nuclear explosions certainly do not represent the best method for performing physics experiments. With the signature of the Total Test Ban Treaty they will be soon be ruled out.

It is possible nowadays to reach very high pressures in the laboratory using shock waves generated by a high-power ing plasma is created on the laser side of the target (the plasma "corona") by focusing high-intensity laser pulses onto solid targets. Owing to momentum conservation, a shock wave travels into the target. Pressures up to 0.75 Gbar have been obtained recently at the Lawrence Livermore Laboratory in the United States [1], and research along the same lines is being carried out in Europe using existing laser systems such as those available at the Laboratoire pour l'Utilisation des Lasers Intenses (LULI) and the Max-PlanckInstitut für Quantenoptik (MPQ). While much more powerful lasers are available in the United States, a large scientific users community has not developed there, partly due to a long-standing classification of inertial confinement fusion data which was only removed in 1993. European Union support that granted LULI, MPQ and other laboratories "large facility" status to allow access by research and university groups to perform experiments has also been an important factor.

\section{High Pressures in Astrophysics}

Ideal-gas physics is usually a good approximation for the rarefied plasmas handled by astrophysicists. But this is unfortunately not always the case. In particular, the density at the centres of stars and giant planets is so high that matter is ionized and becomes a strongly coupled plasma: the various ions interact and no longer behave as free particles. Moreover, pulsed laser beam. A hot, rapidly expand- this behaviour is often accompanied by electron degeneracy as a result of the high electron density. Consequently, the behaviour of stellar and planetary matter exhibits some surprises. For example, a group at Livermore [2] recently used aluminium targets accelerated to about $7 \mathrm{~km} / \mathrm{s}$ by light-gas guns to compress liquid hydrogen to reach the conditions of pressure and temperature comparable to those inside Jupiter. Contrary to what was previously believed, their results seem to suggest that the equation of state of hydrogen at these pressures is such that a sharp boundary does not exist between the planet's core and its mantle. The higher pressures that can be attained with laser beams would yield more accurate information about the planet's interior.

Knowledge of the equation of state is also important for understanding certain aspects of the theory of stellar evolution. For instance, for most white dwarfs, the pressure of degenerate electrons supports the star's material against gravity. But near the star's surface the electrons become less degenerate and ions become important. These ions determine the specific heat and hence the rate at which the white dwarf cools, a process that takes millions of years. So it is necessary to establish the equation of state of the near-surface material in order to understand how white dwarfs evolve.

Brown dwarfs are also poorly understood owing in part to our imperfect knowledge of equations of state. Brown dwarfs have masses so small that their interiors never become hot enough to

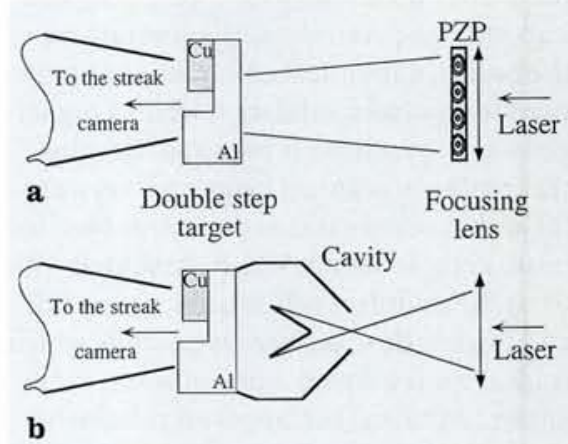

Fig.1. Schematic drawings of the experimental arrangements for direct- and indirect-drive high-pressure laserpulse experiments. A streak camera was used in both cases to measure, shot by shot, the shock velocities in the two-step targets made two materials. a: Directdrive configuration: the laser beam, smoothed with an array of phase zone plates, was focused onto the target. b: Indirect-drive configuration: the laser beam was focused in the cavity whose geometry was designed to avoid preheating. 
burn hydrogen. They simply condense from the interstellar medium and shine dimly for a while as they get rid of the heat of condensation. Their existence, theoretically predicted some time ago, has recently been confirmed experimentally [3], and it is possible that they are so numerous that they comprise half of the mass in our galaxy (the mass which has been inferred to exist from the dynamics of the galaxy, but has not been observed in luminous form). The internal structure of brown dwarfs and their cooling time depend on the details of the equation of state at densities approaching the solid density at a temperature of a few $\mathrm{eV}$ - conditions which are easily attained in laser experiments.

\section{Inertial Confinement Fusion}

Inertial confinement fusion (ICF) is the other important field of physics that relies upon equations of state. In ICF, a thermonuclear target containing deuterium and tritium implodes following illumination by intense laser or ion beams, allowing the conditions for nuclear fusion between $\mathrm{D}$ and $\mathrm{T}$ nuclei to be reached. Gigabar pressures are predicted to arise in spherically compressed capsules. The thermodynamics and hydrodynamics of these systems cannot be analyzed without a knowledge of the equation of state describing how a material reacts to pressure. Moreover, a definitive interpretation of important experiments on the RayleighTaylor instability of imploding ICF capsules [4] requires a knowledge of the equation of state of the targets.

\section{Shock Production with Lasers}

Equation-of-state experiments with shock waves are based on the so-called Hugoniot - Rankine (HR) relations. For the simplest case of "strong" shocks [5]:

$$
\begin{aligned}
& \rho_{0} D=\rho(D-u) \\
& P-P_{0}=\rho_{0} D u \\
& E-E_{0}=u^{2} / 2
\end{aligned}
$$

where $u, \rho, P, D$, and $E$ are the fluid velocity, mass density, pressure, shock velocity, and specific internal energy, respectively, of the material after the passage of a shock wave, while the subscript " 0 " indicates the same parameters for the unperturbed material (i.e., before the shock passage). Being a system of three equations with five unknowns, the Hugoniot - Rankine relations allow a single point on the equation of state of the material to be determined once two parameters have been measured experimentally.
Although it has been well known for many years that lasers can produce shocks with pressures up to $100 \mathrm{Mbar}$ [6], the equation-of-state community has always been reluctant to use laser-generated shocks as a quantitative tool for highpressure physics. It was believed that the quality of laser-generated shocks, as reflected by shock velocities in the material and the planarity of the shock front, was poor. Flat shock fronts and constant shock-front velocities are essential for obtaining accurate estimates of the equation of state. Another, more important, problem is preheating. Hot electrons or hard X-rays produced in the lasergenerated plasma corona penetrate the material ahead of the shock thereby preheating it. In this case, the shock wave travels in a perturbed material and the Hugoniot - Rankine relations are useless.

In recent experiments using two different methods, we have shown that laser pulses can be used to create spatially very uniform shocks in solids with negligible preheating. The first method [7] consists in producing shock waves by direct laser drive using optically smoothed laser beams (see Fig. 1a). The second [8] uses thermal x-rays from laser-heated cavities to generate shocks (indirect laser drive where the laser beam is focused onto a small cavity through an entrance hole see Fig. 1b). Isotropic $x$-ray radiation is created whose temperature depends upon the cavity size and the laser power.

For the generation of intense shocks, direct drive allows higher pressures for the same incident laser energy since no energy is lost in the intermediate step of X-ray conversion. The shock pressures $P$, in Mbar, are given by [9]:

$$
\begin{aligned}
& P_{\mathrm{dir}} \approx 8.6\left(I_{\mathrm{L}} / 10^{14}\right)^{2 / 3} \lambda^{-2 / 3} \\
& P_{\text {ind }} \approx 44\left(I_{\mathrm{C}} / 10^{14}\right)^{10 / 13} \tau_{\mathrm{L}}^{-3 / 26}
\end{aligned}
$$

for direct and indirect drive, respectively, for a primary X-ray flux on the cavity wall $I_{\mathrm{C}}$, a laser intensity on target $I_{\mathrm{L}}$ (both in $\mathrm{W} / \mathrm{cm}^{2}$ ), a laser pulse duration $\tau_{\mathrm{L}}$ (in $\mathrm{ns}$ ), and a laser wavelength $\lambda$ (in $\mu \mathrm{m}$ ). Hence, in order to reach the same pressure, the laser pulse energy required with the indirect method is a factor 2-3 larger than for direct drive for typical laser and cavity parameters.

With regard to the shock quality, the indirect-drive approach has always been considered superior since it assures more uniform irradiation and hence produces smoother and more planar shock waves. Indeed, in direct drive, laser intensity dis-

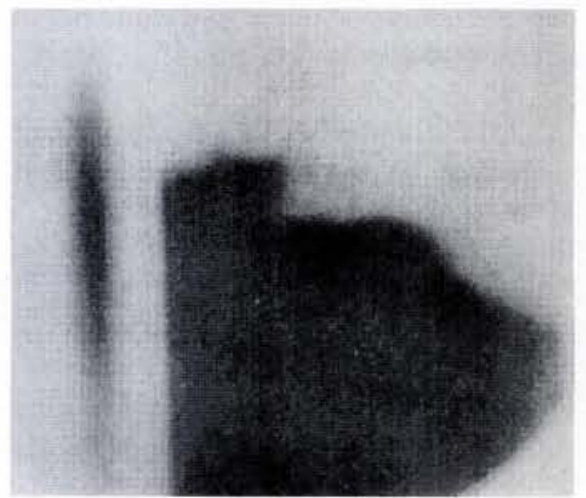

Fig. 2. A streak camera image of the thermal emission from the rear face of one-half of a two-step, twomaterial target at the moment in time when a laserdriven shock broke through (direct drive; aluminium target; $7.5 \mu \mathrm{m}$ base, $2.8 \mu \mathrm{m}$ high step). The horizontal axis represents the distance along the diameter of the focal spot and the vertical axis is the time. The narrow stripe on the left is part of the incident laser beam used as a time fiducial. The difference between the times for shock breakthrough at the base and at the step is 110 $\mathrm{ps}$, corresponding to a shock velocity of $25.5 \mu \mathrm{m} / \mathrm{ns}$. The flat region in the image extends over $180 \mu \mathrm{m}$ so the optically smoothed laser beam gave a remarkably planar shock front.

tribution in the focal spot on the target surface is not flat but, to a first approximation, Gaussian and with many speckles or hot spots. Relatively uniform irradiation can only be achieved using very large focal spots and targets with dimensions smaller than the radius of the focal spot. These requirements of course mean that directdrive experiments call for very high energy lasers or the use of energy smoothing as described here. On the other hand, $\mathrm{X}$-ray preheating is a serious problem for indirect drive owing to the very high $\mathrm{X}$-ray flux produced inside the heated cavity. By optimizing the shape of the cavity we have shown that it is possible to reduce drastically the preheating.

For our direct-drive experiments we used phase zone plates (PZPs) to smooth the beam [10]. PZPs comprise an array of Fresnel lens, where each lens imparts a random dephasing of o or $\pi$ to break down the spatial coherence of the laser beam, thereby giving a smoothing effect similar to that produced by the more common technique of optical smoothing (e.g., that based on random phase plates).

Unlike random phase plates, PZPs allow a "top-hat" intensity distribution to be obtained at the focal spot. As a result, twodimensional effects are almost completely eliminated (around the centre of the focal spot) and high quality, flat shock waves are generated. The technique also allows high pressures (10 - 50 Mbar) to be reached 
over the flat region of the spot with relatively low-power lasers $(\approx 100 \mathrm{~J})$.

\section{Experimental Set-up}

Experiments with laser intensities $I_{\mathrm{L}}$ of $2 \times 10^{14} \mathrm{~W} / \mathrm{cm}^{2}$ were performed using the MPQ's ASTERIX iodine laser and at LULI. The Garching experiments were unusual in that the high laser energy per pulse allowed data to be obtained for both the direct and the indirect methods.

The same diagnostic technique to detect the shock emerging from the rear face of the target was employed in the two configurations. The arrival of the shock at the rear face causes a sudden increase in temperature and hence emissivity. So the shock wave can be monitored by observing the time-dependence of the emissivity, in the visible region, of the rear face using a photographic objective that imaged the face onto the slit of a streak camera.

For the indirect laser-drive configuration we used a $1 \mathrm{~mm}$ in diameter gold cavity and measured the temperature radiation in the $100-150 \mathrm{eV}$ range. The cavity was designed to not only reach these high temperatures, but also to minimize the preheating of the target resulting from the production of primary X-rays. A shield with a conical shape was constructed so that the laser irradiated area and the shocked material were not in direct view of each other, as shown in Fig. 1 b.

Finally, instead of measuring two parameters in order to determine the points on the equation of state, in our experiments we used the impedancematching technique in conjunction with targets made from two materials and having two steps. Each target comprised an aluminium base (the reference material) which supported two steps, one of aluminium and the other made of the material under investigation. This target geometry allows the shock velocities to be determined experimentally in the two materials during the same laser shot. Knowing the equation of state for aluminium and using

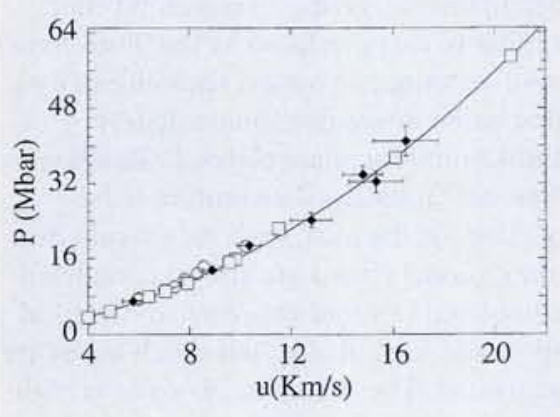

the laws of shock transmission at the interface between the two materials (the impedance matching conditions) one can find points on the relative equation of state for an unknown material. The reliability of this method, which has been used for nuclear experiments, was verified recently for laser-driven shock experiments [11].

\section{Results}

Fig. 2. shows a "flat" shock breakthrough obtained with the PZP beamsmoothing technique and a stepped, twomaterial target. The shock velocity can be determined with high precision by comparing the times at which the shock emerged from the base of the target and from the step.

Fig. 3 gives the entire set of experimental determinations of the relative equation of state for copper in the $10-40$ Mbar pressure range, plotted in the usual pressure $P$ - velocity $u$ coordinates of the impedance-matching technique. The data given in Fig. 3 are usually referred to as the shock polar for the material. Fixing $P$ gives $u$ from the shock polar; substitution of $u$ in the last $\mathrm{HR}$ equation gives the energy $E$; substitution of $u$ and $P$ in the second HR equation gives the shock energy $D$; substitution of $D$ and $u$ in the first HR equation gives the density $\rho$. The outcome is an $(E$, $P, \rho)$ point on the equation of state, where the energy $E$ is an alternative to the temperature $T$ since the two are related.

The equation of state is well defined at pressures below $5 \mathrm{Mbar}$ thanks to measurements performed with gas guns and chemical explosions. The interesting region where there are few data lies above $10 \mathrm{Mbar}$. Our data in this region are plotted together with those of Trunin [12] and Rothman et al. [13] obtained using nuclear explosions and indirect drive, respectively. The solid line gives the $P$ - $u$ relationship calculated using the Los Alamos National Laboratory's SESAME data tables [14].

Error bars were estimated by taking into account all of the sources of error in the measurement of shock velocity, i.e., uncertainties in step thickness, shock breakthrough time, streak-camera sweep speed, etc. They correspond to points for the relative equation of state of copper accurate to better than $\pm 8 \%$ - a level which can be easily improved.

Comparisons with the reported data show that our more efficient direct-drive configuration achieved higher pressures (up to $40 \mathrm{Mbar}$ ). The difference between the direct- and indirect-drive configurations can be appreciated quantitatively by comparing the results obtained at LULI with the indirect-drive results obtained at the MPQ. For the former, pressures in aluminium of $10 \mathrm{Mbar}$ were produced using the direct-drive scheme with a laser energy of $70 \mathrm{~J}$ on the target. With indirect drive, a $250 \mathrm{~J}$ laser energy was needed to reach the same pressure.

The direct-drive method which has been developed can now be used to determine points along the equations of state of other materials of interest in astrophysics (e.g., carbon and iron), and of doped plastics and low-density foams where data is needed in order to analyze the results of some experiments conducted within the framework of inertial confinement fusion research.

\section{References}

[1] R. Cauble et al., Phys. Rev. Lett. 70 (1993) 210 [2] W. Nellis, M. Ross \& N. Holems, Science 269 (1995) 1249.

[3] T. Nakajima et al., Nature 378 (1995) 463; B. Oppenheimer et al., Science 270 (1995) 1478. [4] B. Remington et al., Phys. of Fluids B 5 (1993) 2589.

[5] Ya. Zeldovich \& Yu.Raizer, Physics of Shock Waves \& High Temperature Hydrodynamic Phenomena (Academic Press, New York, 1967).

[6] F. Cottet et al., Appl. Phys. Lett. 47 (1985) 678.

[7] M. Koenig et al., Phys. Rev. E 50 (1994) R3314.

[8] T. Löwer et al., Phys. Rev. Lett. 72 (1994) 3186.

[9] J. Lindl, Phys. Plasma 2 (1995) No.11.

[10] R.M. Stevenson et al., Optics Lett. 19 (1994) 363.

[11] M. Koenig et al., Phys. Rev. Lett. 74 (1995) 2260.

[12] R.F. Trunin, Phys. Uspekhi 37 (1994) 1123.

[13] S.D. Rothman et al., Proc. APS Topical Conf. on Shock Waves, Seattle (1995)

[14] SESAME, Report No. LALP-83-4 ( $\mathrm{T}_{4}$ Group LANL, Los Alamos, 1983).
Fig.3. Shock pressure $P$ as a function of the fluid velocity $u$ for copper. Experimental measurements are compared with the continuous line calculated using data taken from the Los Alamos SESAME tables [14]. The experimental points are for: laser-driven direct- and indirect-drive (solid squares); underground nuclear explosions (open squares) [12] ; indirect drive (open circles) [13]. 